head apparatus was made identical to that of the Pilcon trip-monkey the standard penetration tests were almost identical. The inference is that the one-turn slip-rope method and the Pilcon trip-monkey, other things being equal, give the least impeded fall. The penalty for using two turns in the American Society of Testing and Materials method is to increase the standard penetration test by $40 \%$ due to impedance. This is evidenced by Frydman (1970) and possibly by Gibbs and Holtz (1957) if they followed what appears to be usual practice. The hand-drop, surprisingly, gives a higher standard penetration test than the one-turn sliprope with an equal driven weight, and our conclusion is that it is more difficult to release the weight instantaneously by hand than on the cat head. From the consistency of the results in the tables it is clear that the standard penetration test is quite reproducible. Free fall is much more difficult to achieve than most people imagine and, although a double turn on a cat head seems to clear freely, it does not do so. The trip-monkeys give the same standard penetration test as the one-turn slip-rope method. It is concluded that both comply with the American Society of Testing and Materials. The trip monkeys are thought to be the more reliable because the drop is really free and the height of drop is automatically regulated. Anyone correlating the results of standard penetration tests should first ensure that the tests comply with the American Society of Testing and Materials. A detailed account of our tests has been published (Serota and Lowther, 1973).

\title{
REFERENCES
}

Frydman, S. (1970). Discussion on the dynamic penetration test: a standard that is not standardized. Géotechnique 20, No. 4, 454-455.

Gibbs, H. J. \& Holtz, W. G. (1957). Research on determining the density of sands by spoon penetration testing. Proc. 4th Int. Conf. Soil Mech., London I, 35-39.

Ireland, H. O., Moretto, O. \& Vargas, M. (1970). The dynamic penetration test: a standard that is not standardized. Géotechnique 20, No. 2, 185-192.

Serota, S. \& Lowther, G. (1973). Ground Engineering 6, No. 1, 20-22.

\section{An investigation of a plane strain continuous penetration problem}

BUTTERFIELD, R. and ANDRAWES, K. Z. (1972). Géotechnique 22, No. 4, 597-617.

\section{A. Drescher, Polish Academy of Sciences, Institute of Fundamental Technological Research}

Tests identical to those reported by Butterfield and Andrawes were performed by Drescher et al. (1967) and were aimed at verifying applicability of different flow rules in predicting the velocity field during wedge penetration. A medium-dense sand and an analogue model composed of glass cylinders were used in the experiments. A theoretical analysis of both static and kinematic fields showed fair agreement with the assumption of incompressibility of flow and coaxiality between stress and strain rate tensors. Good agreement was obtained between the theoretical and experimental force-penetration curve although small oscillations of load were also detected.

The Authors' general statements about the advantages of the wedge penetration test are essentially the same as those contained in our paper (1967) but with one important exception. They make a statement about geometric similarity of flow during wedge penetration. This statement is erroneous because the effect of sand weight cannot be neglected. 
In fact, the mesh of stress characteristics changes with increasing depth of penetration and accordingly the velocity characteristics also change. This effect is clearly demonstrated in our paper (1967).

Except for some details on pressure distribution along the wedge and data on flow of high density sand, the other main conclusions of the work by Butterfield and Andrawes coincide with ours. However, our goal was slightly different. It was to provide an accurate analysis of the velocity field and check applicability of assumptions such as incompressibility of flow and coaxiality of tensors occurring in the constitutive law. It seems that for medium-dense sand such assumptions are justified and provide good correlation between experiment and theory, although non-coaxiality has recently been observed in testing the analogous granular material (Drescher and de Josselin de Jong, 1972).

In conclusion I am surprised, seeing these experiments performed with the same motivation for them and with some conclusions the same as those reached in the original work by Drescher et al., that no reference was made to this work.

\section{REFERENCES}

Drescher, A., Kwaszczyńska, K., \& Mróz, Z. (1967). Statics and kinematics of the granular medium in the case of wedge indentation. Archwm Mech. Stosow. 19, No. 1, 99-113.

Drescher, A. \& de Josselin de Jong, G. (1972). Photoelastic verification of a mechanical model for the flow of a granular material. Jnl Mech. Phys. Solids 20, No. 6, 337-351. 